\title{
Modeling of the Investment Program Formation Process of Ecological Management of the Agrarian Cluster
}

\author{
By Mykola Zos-Kior ${ }^{1}$, Olga Shkurupii ${ }^{2}$, Iryna Hnatenko ${ }^{3}$, Oleg Fedirets ${ }^{4}$, \\ Iryna Shulzhenko ${ }^{5}$, Viktoriia Rubezhanska ${ }^{6}$
}

\begin{abstract}
The existence of contradictions between the natural environment and economic activity, including the functioning of agricultural clusters, has led to the need to transfer production to a qualitatively new level, associated with adaptation to environmental conditions. In this regard, the objective of this study is to develop algorithms and economic-mathematical models for environmental safety assessment and decision support in the environmental management system of the agricultural cluster. The article formulates criteria for assessing the environmental safety of agricultural enterprises, which are divided by types of environmental performance of the agricultural cluster (impact on the environment, impact on workers of enterprises participating in the cluster, impact on the environment and people through agricultural cluster products). On the basis of the specified criteria the algorithm of ecological management system estimation of the enterprises-participants of an agrarian cluster is constructed. As a result of the study, an economic and mathematical model of the formation of the investment program of the agricultural cluster was developed, aimed at protecting the environment and improving the environmental situation in general.
\end{abstract}

Keywords: Agricultural Enterprise, Ecological Safety, Investments, Model, Natural resources

\section{Introduction}

In the context of globalization and increasing international competition, the most effective form of organization of agricultural enterprises are clusters, which are sources of economic growth in rural areas by promoting free trade, free movement of capital and human resources, and reducing costs of cluster members through joint technological cooperation (Hnatenko, 2020; Samborskyi, 2020). However, scientific and technological progress has led not only to the emergence of new effective forms of industrial cooperation, but also to increased man-made pressure on nature, as well as to a significant expenditure of non-renewable natural resources. In this situation, the existence of

| ${ }^{1}$ Dr. Sc., Associate Professor, Educational and Scientific Institute of Economics, Management, Law and Information Technology, Poltava State Agrarian Academy, Ukraine

${ }^{2}$ Dr. Sc., Professor, Educational and Scientific Institute of Economics, Management, Law and Information Technology, Poltava State Agrarian Academy, Ukraine

${ }^{3} \mathrm{PhD}$, Associate Professor, Faculty of Entrepreneurship and Law, Kyiv National University of Technologies and Design, Ukraine

${ }^{4} \mathrm{PhD}$, Associate Professor, Educational and Scientific Institute of Economics, Management, Law and Information Technology, Poltava State Agrarian Academy, Ukraine

${ }^{5} \mathrm{PhD}$, Associate Professor, Educational and Scientific Institute of Economics, Management, Law and Information Technology, Poltava State Agrarian Academy, Ukraine

${ }^{6} \mathrm{PhD}$, Educational and Research Institute of Economics and Business, Luhansk Taras Shevchenko National University, Ukraine (Corresponding author). 
contradictions between the environment and economic activity, including the functioning of agricultural clusters, has led to the need to transfer production to a qualitatively new level, associated with adaptation to environmental conditions. Achieving this level is possible with the development of new management methods that provide the relationship between anthropogenic and natural ecological systems, aimed at harmonizing the laws of ecology and economics. That is, ensuring sustainable development of agriculture is directly dependent on the level of greening of its production. At the same time, it is very important to maintain a balance between environmental safety, which requires significant financial costs, and economic efficiency of the business entity. In such a situation, it is necessary to organize production activities in such a way that it provides a reasonable compromise between production goals and their environmental consequences. One of the ways to solve this problem is to develop economic and mathematical models to support management decisions in the field of environmental management.

The work of many scientists is devoted to the study of various aspects of environmental safety of economic entities, including enterprises participating in agricultural clusters, as well as to the modeling of management processes in the field of environmental management. Thus, Ramirez et al. (2018) analyzed intermediary organizations in agricultural clusters, identified their impact on the formation of environmental safety of clusters. Puppim de Oliveira and Jabbour (2017) found that the use of three types of cluster management in agriculture will help improve the environment, namely: the use of law, supply chain pressure and the voluntary use of the concept of corporate social responsibility (CSR). The scientific work of Kearins et al. (2010) examines the growth of enterprise potential through the effective implementation of environmental management measures. Ormazabal et al. (2017) developed a model of the evolution of environmental management in the enterprise based on the definition of the stages of the company maturity and the factors influencing the formation of these stages. Tang et al. (2015) examined the policy framework for environmental management in China and Taiwan and identified the role of the public in assessing the environmental impact of enterprises. Chaikin et al. (2018) conducted socio-economic monitoring and proposed recommendations for the introduction of environmental management systems in agricultural enterprises of Ukraine. Melnyk and Tsesarsky (2019) consider specific aspects of environmental safety as a principle of management of agricultural enterprises, indirectly determined by international legal standards. $\mathrm{Li}$ et al. (2020) developed a decision-making system in the field of coordinating the development of land use models in agriculture based on the use of specific methods of environmental management. Kruse et al. (2019) proposed an integrated structure of the system of lean management, as well as strategies for the simultaneous protection of workers, the environment and support of certain financial results achieved by the agricultural enterprise. The article by Buffa et al. (2018) examines the practical measures of environmental management used by small and medium-sized Italian companies to stimulate the development of sustainable business models. The research of Dey et al. (2018) is devoted to the development of recommendations for optimizing the implementation of small and medium-sized enterprises (SME) in the agricultural sector of the economy, practical measures of environmental management and corporate social responsibility. Based on bibliometric analysis and systematic review of scientific articles on the implementation of 
environmental management in SME, Ferenhof et al. (2014) identified the basic aspects of the environmental management system most often used by these enterprises in business, namely: certification, fault analysis and implementation of improvements, environmental responsibility, environmental mitigation. Hang and Chunguang (2015) investigated the nature of the impact of environmental management on the value of the agricultural enterprise and found that within the specific measures of environmental management, the production of environmentally friendly products will significantly increase corporate value. Armas-Cruz (2011) identifies the determinants and implications of environmental management in Spanish companies and confirms the positive impact of the use of environmental management measures on the company's economic performance.

Paying tribute to the above scientific works, it should be noted the need for further research in the direction of economic and mathematical modeling of processes in the field of environmental management of agricultural clusters. In this regard, the objectives of this article are: 1) to determine the criteria for assessing the environmental safety of agricultural enterprises in the cluster; 2) formation of an algorithm for evaluating the environmental management system of enterprises participating in the agricultural cluster; 3) development of economic and mathematical model of the investment program formation of the agrarian cluster, aimed at environmental protection and ecological situation improvement.

\section{Materials and Methods}

One of the sources of the environment pollution and agricultural lands are agricultural enterprises, warehouses for storage of mineral fertilizers and pesticides, intensification of agricultural production chemicalization. Thus, in Ukraine, according to the State Statistics Service of Ukraine (2019), during 2014-2018 there is a significant increase in the level of pesticide use (in the active substance) for crop yields (Figure. 1).

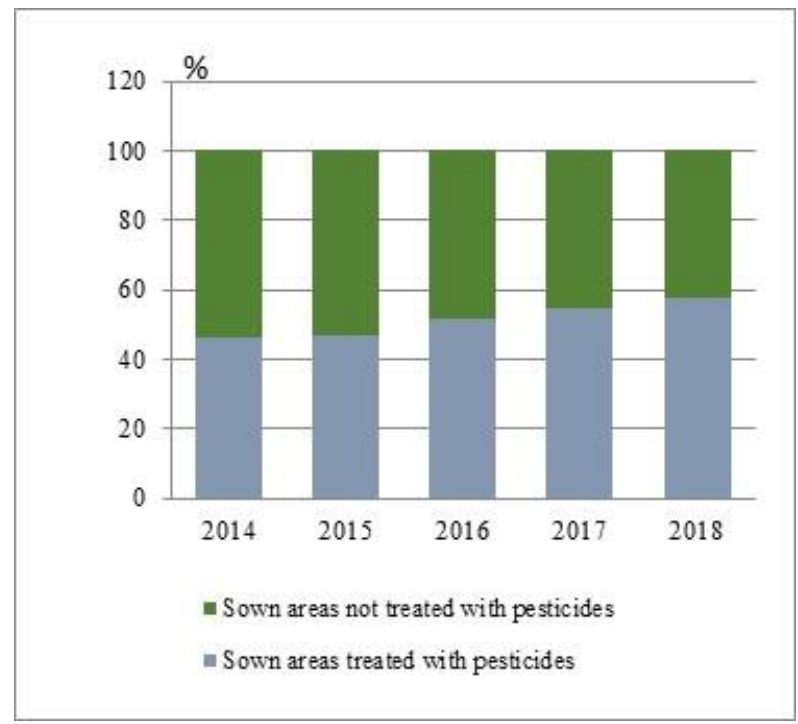

Figure. 1: Dynamics of the pesticide treatment level of crops sown areas for the harvest of 2014-2018 in Ukraine 
At the same time, there is a positive trend to reduce the amount of waste from economic activities of agricultural enterprises (Figure. 2).

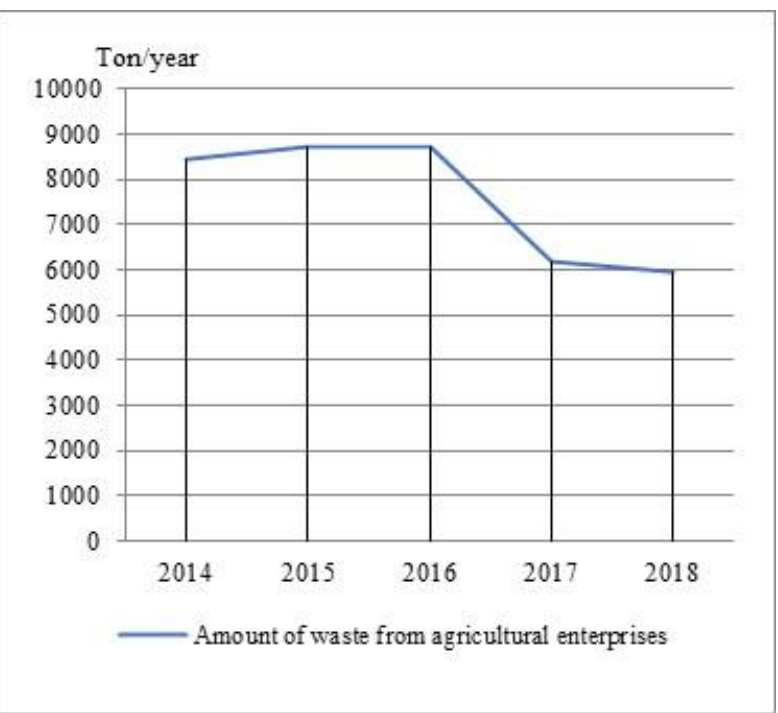

Figure. 2: Dynamics of waste generation from agricultural enterprises in Ukraine during 2014-2018

Currently in Ukraine there are no official statistics on the accounting of key performance indicators of agro-industrial clusters. Given the deterioration of the environmental situation in Ukraine and in order to optimize the production activities of enterprises participating in the above agricultural clusters, it is considered to be necessary to conduct a comprehensive assessment of environmental safety of these entities. The system of criteria for this assessment should cover all levels of interaction of enterprises participating in the agricultural cluster with the environment and be based on the existing regulatory and information base (Goncharov et al., 2013). Otherwise, due to lack, absence or unrepresentativeness of the source information, practical calculations of the proposed indicators will be impossible. Thus, the set of characteristics and indicators of environmental safety of enterprises participating in the agricultural cluster should provide: assessment of the enterprise safety level in the conditions of normal operation (all three main aspects of functioning - ecological, social and economic should be covered); forecast of security level in case of enterprise modernization or change of its structure; assessment of enterprise resource consumption; assessment of the accidents probability and hazards in emergency conditions. The last point is more related to the technological level of security.

Based on the above requirements, a tree of criteria for assessing the environmental safety of enterprises participating in the agricultural cluster has been developed, shown in Figure. 3. 


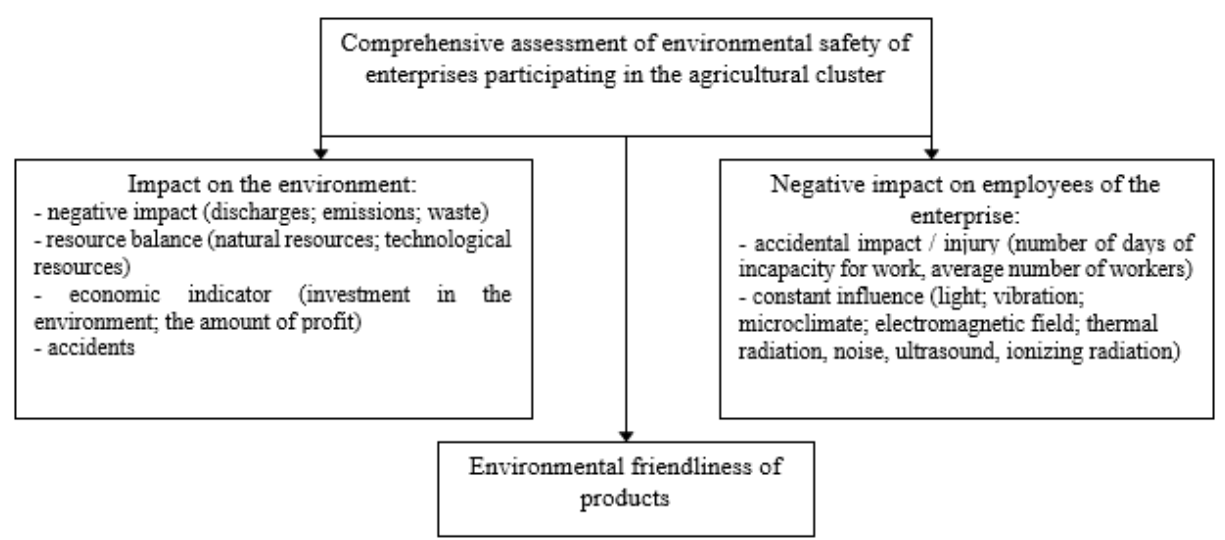

Figure. 3: Architectonics of criteria for assessing the environmental safety of enterprises participating in the agricultural cluster

The main criteria for assessing the environmental safety of agricultural enterprises will be considered in more detail. Thus, the indicator of impact on the environment determines the level of danger of this impact. It is represented by the following groups of indicators: negative impact, resource balance and economic indicators.

The negative impact is represented by air emissions, industrial wastewater discharges and industrial waste. All three indicators are calculated similarly, and accordingly consist of similar components: the maximum allowable exposure rate, the hazard class of the substance or waste, the level of actual impact for the current period and the level of actual impact for the same past period.

The resource balance is presented as the difference between the amount consumed and the amount of reproduced individual resource. It should be noted that, since enterprises are mainly consumers of resources, in the analysis of environmental safety resource balances are usually negative.

With the help of economic indicators, the level of enterprise costs for environmental activities is determined. The costs of the enterprise are presented in the form of the ratio of investments and environmental payments. Environmental payments are, in fact, derivatives of specific losses. However, when determining payments: first, it takes into account the excess of the company's permissible standards of influence (limit and excess payments, or fines); secondly, payments for the limit and over-limit use of natural resources allow to some extent to assess the resource component of environmental safety; thirdly, environmental payments are regulated by a single system of laws and regulations and are mandatory for each company, i.e. to obtain them it is enough to raise the relevant financial statements of the company.

The investment ratio is a percentage of the company's profits, which is directed to measures to protect the environment and improve environmental safety.

The indicator "Accidents at the enterprise" is determined by the number of accidents during the analyzed period.

The group of criteria for negative impact on employees of the enterprise is represented by indicators of injuries at the enterprise, which occurs accidentally, and indicators of constant 
impact on employees of the enterprise such as noise, vibration, ultrasound and other factors affecting the health of employees and related to occupational diseases. These indicators consider the values of the maximum allowable level of impact, the actual level of impact for the analyzed period and the actual level of impact for the same past period. Environmental friendliness of products is the properties of products that determine the harmful effects on the environment during production, storage and disposal. We propose to determine the environmental friendliness of products by direct expert evaluation on a five-point scale (1-extremely dangerous, 2-highly dangerous, 3-moderately dangerous, 4slightly dangerous, 5 -safe).

During the assessment, the final comprehensive indicator of environmental safety of the enterprise $\left(Y_{e s}\right)$ can take the following values (on a five-point scale): 1 - extremely dangerous; 2 - highly dangerous; 3 - moderately dangerous; 4 - slightly dangerous; 5 - safe. The value of the final indicator $Y_{e s}$ depends on the values of the indicators of the previous level of the hierarchy (1):

$Y_{e s}=f\left(Y_{e i}, Y_{e f}, Y_{\text {nie }}\right)$,

where $Y_{e i}$ - a comprehensive indicator of the company's impact on the environment;

$Y_{f f}$ - an indicator that characterizes the environmental friendliness of the enterprise;

$Y_{\text {mie }}$ - a comprehensive indicator of the negative impact on employees of the enterprise.

Estimation of $Y_{e i}$ similarly to a complex indicator of ecological safety is measured on a five-point scale and can accept value: 1 - extremely dangerous; 2 - highly dangerous; 3 moderately dangerous; 4 - little dangerous; 5 - safe.

The value of $Y_{e i}$ depends on the values of the indicators of the previous level of the hierarchy (2):

$Y_{e i}=f\left(Y_{n i}, Y_{r b}, Y_{i n v . e m}, Y_{a}\right)$,

where $Y_{n i}$ - complex indicator of negative impact (discharges, emissions, wastes) on the natural environment;

$Y_{r b}$ - indicator of the enterprise resource balance,

$Y_{\text {inn.em }}$ - an indicator that characterizes the economic activity of the enterprise (investment in environmental protection),

$Y_{a}$ - an indicator that characterizes the damage to the environment from accidents.

Resource balance $\left(Y_{r b}\right)$ is calculated as the difference between produced resources $\left(R_{p r}\right)$ and resources consumed in the production process $\left(R_{c}\right)(3)$ :

$Y_{r b}=R_{p r}-R_{c}$

The economic indicator (investment ratio - $Y_{\text {inn.em) }}$ ) shows the share (as a percentage) of investments directed to environmental measures in the company's profit (4):

$Y_{\text {invem }}=\operatorname{Inv} /$ Prof * 100,

where Inv - amount of investment;

Prof-profit.

It can take the following values: no investment (score 1); $1 \%-5 \%$ (score 2); $5 \%-15 \%$ (score 3); $15 \%-25 \%$ (score 4); more than 25\% (score 5).

Accidents $\left(Y_{a}\right)$ are calculated based on Eq. 5:

$Y_{a}=N_{a} / T * 365$,

where $N_{a}$ - the number of accidents during the analyzed period $T$.

We will assume that if $Y_{a}=0$, then the accident rating corresponds to the value 5 "safe", 
if $Y_{a}<0,1$ - score 4 (slightly dangerous), if $0,1<Y_{a}<2$ - score 3 (moderately dangerous), if $2<Y_{a}<5$ - grade 2 (highly dangerous), if $Y_{a}>5$ - grade 1 (extremely dangerous).

The complex indicator of negative impact $\left(Y_{n i}\right)$ is calculated based on the Eq. 6:

$Y_{n i}=($ Emissions + Discharges $+W$ aste $) / 3$,

Depending on the value obtained by Eq. 6, the considered indicator acquires the following values: more than 2 - extremely dangerous (grade 1); 1.51-2 - highly dangerous (score 2); 1.21-1.5 - moderately dangerous (score 3); 1.01-1.2 - slightly dangerous (score 4); to 1 safe (score 5).

An indicator that characterizes the environmental friendliness of the enterprise $\left(Y_{e f}\right)$ is determined by direct expert evaluation and can take values from 1 to 5: 1 - extremely dangerous; 2 - highly dangerous; 3 - moderately dangerous; 4 - slightly dangerous; 5 - safe. When assessing environmental safety, it is necessary to take into account the negative effects on employees $\left(Y_{\text {nie }}\right)$, which are divided into constant effects $Y_{\text {const.e }}$ (working air quality, heat, noise, vibration, ultrasound, light, electromagnetic field, ionizing radiation in the workplace), which have a negative effects on the health of workers and lead to occupational diseases, and accidental negative effects $Y_{\text {acc. }}$ (injuries).

Indicators of the first group (permanent impact) are calculated as the ratio of the actual level (Fact) to the maximum allowable level (MAL), for example, the noise figure is calculated by the Eq. 7:

Noise = Fact $/ M A L$

Other indicators included in this group are calculated similarly to noise.

When calculating the complex indicator "Constant effects " ( $\left.Y_{\text {const. }}\right)$ the Eq. 8 is used:

$Y_{\text {const.e }}=($ Noise + Vibration $+\ldots+$ Ultrasound $) / N_{\text {per. }}$

where $N_{\text {perf }}$ - the number of persistent negative factors.

As a result, the indicator $Y_{\text {const. }}$ can take the following values: more than 1.3 - extremely dangerous (score 1); 1.21 -1.3 - highly dangerous (score 2); 1.11-1.2 - moderately dangerous (score 3); 1.01-1.1 - little dangerous (score 4); to 1 - safe (score 5).

The following indicators are used in the analysis of injuries $\left(Y_{\text {acc.e }}\right)$ :

Injury frequency ratio $\left(R_{\text {inj.fr }}\right)(9)$ :

$R_{\text {inj:fr }}=\left(N_{a} * 1000\right) / C$,

where $N_{a}$ - number of accidents;

$C$ - average list composition of the enterprise.

Injury severity ratio $\left(R_{\text {inj.sev }}\right)(10)$ :

$R_{\text {inj.sev }}=N_{d} / N_{a}$,

where $N_{d}$ - the number of days of incapacity for work due to an accident.

Coefficient of general injury $\left(R_{\text {inj:gen }}\right)(11)$ :

$\mathrm{R}_{\text {inj.gen }}=\mathrm{R}_{\text {inj.fr }} * \mathrm{R}_{\text {inj.sev }}$

Depending on the value of $R_{\text {inj.gen }}$ injury rate $Y_{\text {acc. }}$ can take three values: 1 - dangerous production $\left(R_{i n j \text { gen }}>40\right) ; 2$ - moderately dangerous $\left(20<R_{\text {inj.gen }}<40\right) ; 3$ - safe $\left(R_{i n j . g e n}<20\right)$.

\section{Results and Discussions}

\subsection{Algorithm for evaluating the environmental management system of enterprises participating in the agricultural cluster}

Since ensuring environmental safety is the main task of environmental 
management, the assessment of environmental safety can serve as an assessment of environmental management itself, conducted according to the above calculation algorithm.

Initially, the value of environmental management assessment $Y_{\text {em }}$ in the reporting period is considered equal to the assessment of environmental safety: $Y_{e m}=Y_{e s}$.

If $Y_{e s} \neq Y_{e s}^{b}$, than environmental management assessment $Y_{e m}$ in the reporting period is specified by the Eq. 12:

$Y_{e m}=Y_{e m}+\left(Y_{e s}-Y_{e s}^{b}\right)$,

where $Y_{e s}^{b}$ - the value of a comprehensive indicator of environmental safety in the base period with which the report is compared.

Further refinement of the assessment of $Y_{\mathrm{em}}$ is not performed.

If the values $Y_{e s}$ and $Y_{e s}^{b}$ are equal, then to account for the dynamics of environmental safety for the reporting period, changes are considered (for the reporting period) in the areas: "Impact on the environment", "Impact on employees", " Environmental friendliness of products ".

Accounting for changes in the direction of "Impact on the environment".

If $Y_{e i} \neq Y_{e i}^{b}$, than environmental management assessment $Y_{e m}$ in the reporting period is specified by the Eq. 13:

$Y_{e m}=Y_{e m}+\left(Y_{e i}-Y_{e i}^{b}\right) / 3$,

Further clarification of the assessment in the section "Impact on the environment" is not carried out.

If $Y_{e i} \neq Y_{e i}^{b}$, then changes for the reporting period are considered in the areas: "Negative impact (discharges, emissions, waste)", "Investment in environmental measures" and "Accidents".

Accounting for changes in the section "Negative effects" is carried out according to the Eq. 14:

$Y_{e m}=Y_{e m}+\left(Y_{n i}-Y_{n i}^{b}\right) / 9$

Further refinement of the assessment under the section "Negative effects" is not carried out.

Accounting for changes in the section "Investments in environmental measures" is carried out according to the Eq. 15:

$Y_{\text {em }}=Y_{\text {em }}+\left(Y_{\text {inv.em }}-Y_{\text {inv.em }}^{b}\right) / 9$

Accounting for changes in the section "Accidents" is carried out according to the Eq. 16:

$Y_{e m}=Y_{e m}+\left(Y_{a}-Y_{a}^{b}\right) / 9$

Accounting for changes in the direction of "Impact on employees of the enterprise".

If $Y_{\text {imp.em }} \neq Y_{i m p . e m}^{b}$, than environmental management assessment $Y_{\text {em }}$ in the reporting period is specified by the Eq. 17:

$Y_{\text {em }}=Y_{\text {em }}+\left(Y_{\text {nie }}-Y_{\text {nie }}^{b}\right) / 3$

Further clarification of the assessment under the section "Impact on employees of the enterprise" is not carried out.

If $Y_{\text {nie }}=Y_{\text {nie }}^{b}$, then changes for the reporting period in the directions: "Constant influences (light, vibration, noise, etc.)" and "Trauma" are considered.

Accounting for changes in the section "Permanent impact" is carried out according to the 
Eq. 18:

$Y_{\text {em }}=Y_{\text {em }}+\left(Y_{p i}-Y_{p i}^{b}\right) / 6$

Further clarification of the assessment under the section "Permanent impact" is not carried out.

Accounting for changes in the section "Injuries" is carried out according to the Eq. 19:

$Y_{e m}=Y_{e m}+\left(Y_{i n j}-Y_{i n j}^{b}\right) / 6$

The account of changes in the direction of "Environmental friendliness of production" is carried out by the Eq. 20:

$Y_{e m}=Y_{e m}+\left(Y_{e f}-Y_{e f}^{b}\right) / 3$

Thus, the general model of environmental management assessment for the reporting period is as follows (21):

$Y_{e m}=Y_{e s}+\left(Y_{e s}-Y_{e s}^{b}\right)+k_{1}\left(\left(Y_{e i}-Y_{e i}^{b}\right) / 3+k_{2}\left(\left(Y_{n e}-Y_{n e}^{b}\right) / 9+\left(Y_{i n v . e m}-\right.\right.\right.$ $\left.\left.Y_{i n v . e m}^{b}\right) / 9+\left(Y_{a}-Y_{a}^{b}\right) / 9\right)+\left(Y_{\text {nie }}-Y_{\text {nie }}^{b}\right) / 3+k_{3}\left(\left(Y_{p i}-Y_{p i}^{b}\right) / 6+\left(Y_{\text {inj }}-Y_{\text {inj }}^{b}\right) /\right.$

$\left.6+\left(Y_{e f}-Y_{e f}^{b}\right) / 3\right)$

In this model:

$k_{1}=1$, if $Y_{e s}=Y_{e s}^{b} ; k_{1}=0$, if $Y_{e s} \neq Y_{e s}^{b}$;

$k_{2}=1$, if $Y_{e i}=Y_{e i}^{b} ; k_{2}=0$, if $Y_{e i} \neq Y_{e i}^{b}$;

$k_{3}=1$, if $Y_{\text {nie }}=Y_{\text {nie }}^{b} ; k_{3}=0$, if $Y_{\text {nie }} \neq Y_{\text {nie }}^{b}$;

Note that the presented model of environmental management assessment assumes that the weight values of environmental indicators of one level of the hierarchy are equal to each other. Consider the case where the weight values of the indicators are different.

Let's mark:

$W_{e s}, W_{e f}, W_{n i e}$ - weight values of indicators of the 4th level $Y_{e s}, Y_{e f}, Y_{\text {nie }}$ respectively, $\left(W_{e s}+\right.$ $\left.W_{e f}+W_{n i e}=1\right)$;

$W_{n e}, W_{\text {invem }}, W_{a}$ - weight values of indicators of the 3 rd level $Y_{n e}, Y_{\text {invem }}, Y_{a}$ respectively, $\left(W_{n e}+W_{i n v e m}+W_{a}=1\right)$;

$W_{p i}, W_{i n j}$ - weight values of indicators of the 3 rd level $Y_{p i}, Y_{i n j}$ respectively, $\left(W_{p i}+W_{i n j}=1\right)$.

Then the model of environmental management assessment for the reporting period will look like (22):

$Y_{e m}=Y_{e s}+\left(Y_{e s}-Y_{e s}^{b}\right)+k_{1}\left(W_{e i}\left(Y_{e i}-Y_{e i}^{b}\right)+k_{2} W_{e i}\left(W_{n e}\left(Y_{n e}-Y_{n e}^{b}\right)+\right.\right.$ $\left.W_{\text {inv.em }}\left(Y_{\text {inv.em }}-Y_{\text {inv.em }}^{b}\right)+W_{a}\left(Y_{a}-Y_{a}^{b}\right)\right)+W_{\text {nie }}\left(Y_{\text {nie }}-Y_{\text {nie }}^{b}\right)+$

$k_{3} W_{n i e}\left(W_{p i}\left(Y_{p i}-Y_{p i}^{b}\right)+W_{i n j}\left(Y_{i n j}-Y_{i n j}^{b}\right)+W_{e f}\left(Y_{e f}-Y_{e f}^{b}\right)\right)$

The values of $k_{1}, k_{2}, k_{3}$ are calculated similarly to the corresponding indicators of model (21).

\subsection{Economic and mathematical modeling of the formation of the investment program of the agricultural cluster}

In order to protect the environment and improve the environmental situation, we will develop a conceptual model of income and expenditure planning and resource management, and based on it a model of investment program formation aimed at optimizing the economic activity of the agricultural cluster as a whole.

Thus, suppose that the studied agricultural cluster consists of agricultural enterprises - 
business units $(\mathrm{BU})$, which directly produce the final products of the cluster, ie with $\mathrm{BU}$, are "profit centers". At the stage of strategic planning, each BU proposes for approval several variants of its business plan, for example, the "minimum option" focuses only on the own resources of the $\mathrm{BU}$, and the "maximum" option involves the use of common resources of the cluster. Let's also assume that a long-term business development plan is drawn up for several planning periods (for example, for three years with a breakdown by quarters).

We introduce the following notation of the mathematical model:

$i$ - index $\mathrm{BU}, i=\overline{1, m}$;

$t, w$ - indices of planning periods, $t=\overline{1, v ;} w=\overline{1, v \text {; }}$

$k$ index of the plan variant $i$-th $\mathrm{BU}, k=\overline{1, s}$; The following parameters are known for each $i$-th $\mathrm{BU}$ for each variant of the business plan:

$O_{i}$ - balance of funds at the beginning of the period;

$A_{\text {itt' }}$ - costs of the main activity in the $t$-planning period according to the $k$-th option;

$A_{\text {itte- }}$ - costs for the main activity cumulatively for $t$-planning periods for the $k$-th option;

$A_{i t k}=\sum_{h=1}^{t} A_{i h k}^{\prime}$;

$B_{i t k^{\prime}}$ - investment costs in the $t$-planning period according to the $k$-th option;

$B_{i t t}$ - investment costs for t planning periods for the $k$-th option;

$C_{i t k^{\prime}}-$ consumption costs and other costs in the $t$-planning period according to the $k$-th option;

$C_{i t k}$ - consumption costs and other costs for $t$-planning periods for the k-th option;

$D_{i t k^{\prime}}$ - income in the $t$-planning period according to the $k$-th option;

$D_{i t k}$ - income for $t$-planning period according to the $k$-th option.

The initial balance of $O_{i}$ is known in the main company of the cluster.

Controlled variables (unknown):

$X_{i t}{ }^{\prime}$ - subsidy of the parent company of the $i$-th $\mathrm{BU}$ in the $t$-planning period;

$X_{i t}$ - subsidy of the parent company of the $i$-th BU cumulative total for $t$-planning periods;

$Y_{i t^{\prime}}$ - raising funds (outflow from the $i$-th BU to the main company) in the $t$-planning period;

$Y_{i t}$ - raising funds (outflow from the $i$-th BU to the parent company) for $t$-planning periods.

$Z_{i k}=1$, if for the $i$-th subdivision on the k-th option is accepted;

$Z_{i k}=0$, if for the $i$-th subdivision on the k-th option is not accepted;

The system of constraints of the mathematical model will look like (23):

$O_{i}+\sum_{k=1}^{s} \sum_{t=1}^{w}\left(D_{i t k} * Z_{i k}\right)+\sum_{t=1}^{w} X_{i t} \geq \sum_{k=1}^{s} \sum_{t=1}^{w}\left(A_{i t k} * Z_{i k}+B_{i t k} * Z_{i k}+C_{i t k} *\right.$ $\left.Z_{i k}\right)+\sum_{t=1}^{w} Y_{i t}, i=\overline{1, m ;} w=\overline{1, v} ; \quad O+\sum_{t=1}^{w} Y_{i t}-\sum_{t=1}^{w} X_{i t} \geq 0, i=\overline{1, m} ; i=\overline{1, v}$; $\sum_{k=1}^{s} Z_{i k}=1, i=\overline{1, m}$

As a function of the goal, depending on the strategic goals can be used: maximizing profitability for the entire period or at the end of the strategic planning period; maximizing the total profit of the cluster for the entire period or at the end of the strategic planning period; maximization of commodity output at the end of the period, etc.

For example, if the maximization of the total profit of the cluster for the entire planning period is taken as a function of the goal, the target function will look like (24):

$F=\sum_{i=1}^{m} \sum_{t=1}^{v} \sum_{k=1}^{S}\left(D_{i t k}^{\prime}\right) /(1+r)^{t} * Z_{i k}-\sum_{i=1}^{m} \sum_{t=1}^{v} \sum_{k=1}^{S}\left(A_{i t k}^{\prime}+B_{i t k}^{\prime}+C_{i t k}^{\prime}\right) / /$ 
$(1+r)^{t} * Z_{i k}+\sum_{i=1}^{m} \sum_{t=1}^{v} Y_{i t} /(1+r)^{t}-\sum_{i=1}^{m} \sum_{t=1}^{v} X_{i t}^{\prime} /(1+r)^{t} \rightarrow \max$, where $r$ - the value of the annual interest rate (to take into account discounting costs) and it is assumed that the planning period is equal to one year.

In order to form a plan of measures (investment program) of the agricultural cluster, aimed at improving the environmental safety of its operation, let's modify the above economic and mathematical model. Thus, let the studied agricultural cluster consist of $n$ business units (enterprises, productions, shops) that have a negative impact on the environmental situation. Each business unit offers measures to improve the environmental safety of its activities. From the main budget of the cluster, a certain amount is planned to increase environmental safety, which should be distributed between enterprises (BU) and the proposed measures.

We introduce the following notation of the mathematical model:

$i$ - index of the enterprise (BU), $i=\overline{1, n \text {; }}$

$j_{i}$ - index of the event (within the $i$-th enterprise) to improve the environmental situation, $j=\overline{1, m_{i}}$

$m_{i}$ - the number of possible measures for the $i$-th enterprise;

$c_{i j}$ - costs for the $i$-th event on the $j$-th enterprise;

$u_{i}$ - the amount of own funds of the $i$-th enterprise, allocated for the environment;

$k$ - index of the type of environmental pollutant, $k=\overline{1, r}$;

$r$ - total amount of pollutants;

$p_{k}$ - the proportion of pollution reduction on the $k$-th indicator of pollution; $\sum p_{k}=1$;

$e_{i j k}=$ effect (for example, reduction of emission of the $k$-th pollutant as a result of the $j$-th measure at the $i$-th enterprise);

$Z_{i j}=1$, if the $j$-th measure on the $i$-th enterprise is included in the plan;

$Z_{i j}=0$, if the $j$-th measure on the $i$-th enterprise is not included in the plan.

$S$ - the total amount of funds allocated to the environment by the main business structure; $x_{i}$ - the amount of funds of the main business structure allocated to the $i$-th enterprise.

Then the economic and mathematical model of the optimal distribution of resources of the agricultural cluster for measures to improve its environmental safety will look like (25): $\sum_{i=1}^{n} \sum_{j=1}^{m_{i}} Z_{i k} \sum_{k=1}^{r} p_{k} e_{i j k} \rightarrow \max \sum_{j=1}^{m_{i}} c_{i j} z_{i j} \leq x_{i}+u_{i}, i=\overline{1, n} \sum_{i=1}^{n} x_{i} \leq S$

First, the basic value of the complex indicator of environmental safety of the cluster $Y_{e s}$ (according to Eq.1-11) in the analyzed period in the absence of any measures to protect the environment and improve the environmental situation. Then for each option the value of the same indicator is determined, if to implement the considered measure in the absence of other measures.

Denote by $Y_{e s}(i j)$ - the value of a comprehensive indicator of environmental safety in the implementation of the $j$-th measure for the $i$-th enterprise and the absence of other measures. Then the effect of the implementation of the $j$-th measure on the $i$-th enterprise will be (26):

$e_{i j}=Y_{e s}(i j)-Y_{e s}$,

and the model of optimal formation of investments in environmental protection and improvement of the ecological situation will be as follows (27): 


$$
\begin{aligned}
& \sum_{i=1}^{n} \sum_{j=1}^{m_{i}} z_{i j} e_{i j} \rightarrow \max \\
& \sum_{\substack{m_{i} \\
j=1}}^{n} c_{i j} z_{i j} \leq x_{i}+u_{i}, i=\overline{1, n} \\
& \sum_{i=1}^{n} x_{i} \leq S
\end{aligned}
$$

In this case, in the model (27):

$c_{i j}$ - the necessary costs for the $i$-th event for the $j$-th enterprise;

$u_{i}$ - the amount of own funds of the i-th enterprise, allocated for the environment;

$S$ - the total amount of funds allocated for the environment from the total budget of the agricultural cluster;

$x_{i}$ - the amount of the general budget allocated to the $i$-th enterprise;

$Z_{i j}=1$, if the $j$-th measure on the $i$-th enterprise is included in the investment program;

$Z_{i j}=0$, if the $j$-th measure on the $i$-th enterprise is not included in the investment program.

Thus, the use in practice of agricultural clusters of the proposed economic and mathematical model of investment environmental program will determine the optimal environmental management measures for a particular cluster enterprise and the amount of financial resources required for this.

\section{Conclusions}

As a result of the study, the positive dynamics in reducing the generation of waste from agricultural enterprises and negative trends in the level of pesticide treatment of sown areas of crops for harvest in Ukraine during 2014-2018. In order to improve the environmental situation, the criteria for assessing the environmental safety of agricultural enterprises are formulated, which are distributed according to the types of environmental performance of the agricultural cluster: impact on the environment (negative impact; resource balance; economic performance; accidents), negative impact on cluster workers (accidental influence; constant influence), environmental friendliness of products. Based on these criteria, a system of comprehensive environmental safety assessment and an algorithm for assessing the quality of environmental management of enterprises participating in the agricultural cluster, which takes into account changes in the environmental situation during the reporting period. Also a conceptual model of income and expenditure planning and resource management was developed. On its basis economic and mathematical model of investment program was generated, aimed at optimizing the economic activity of the agricultural cluster as a whole, which will determine the optimal environmental management measures for a particular cluster member and the amount required for this financial resources.

\section{References}

Armas-Cruz, Y. (2011). Determinants of environmental management and implications for firms' economic performance. Journal of Environmental Planning and Management, 54(8), 1077-1106. 
Buffa, F., Franch, M. and Rizio, D. (2018). Environmental management practices for sustainable business models in small and medium sized hotel enterprises. Journal of Cleaner Production, 194, 656-664.

Chaikin, O., Kirieieva, E. and Slobodeniuk, O. (2018). Environmental management certification: social and economic monitoring. Management Theory and Studies for Rural Business and Infrastructure Development, 40(3), 297-306.

Dey, Pr., Petridis, N., Petridis, K., Malesios, Ch., Nixon, J. and Ghosh, S. (2018). Environmental management and corporate social responsibility practices of small and medium-sized enterprises. Journal of Cleaner Production, 195, 687-702.

Ferenhof, H., Vignochi, L., Selig, P., Lezana, Ál. and Campos, L. (2014). Environmental management systems in small and medium-sized enterprises: an analysis and systematic review. Journal of Cleaner Production, $74,44-53$.

Goncharov, V.M., Zos-Kior, M., Rakhmetulina Z. (2013). The investment component of ukrainian agrarian enterprises' development in conditions of land reform, Actual Problems of Economics, 10 (148), 118 125.

Hang, S. and Chunguang, Zh. (2015). Does environmental management improve enterprise's value? - An empirical research based on Chinese listed companies. Ecological Indicators, 51, 191-196.

Hnatenko, I., Orlova-Kurilova, O., Shtuler, I., Serzhanov, V. and Rubezhanska, V. (2020). An Approach to Innovation Potential Evaluation as a Means of Enterprise Management Improving. International Journal of Supply and Operations Management, 7(1), 112-118.

Kearins, K., Collins, E. and Tregidga, H. (2010). Beyond Corporate Environmental Management to a Consideration of Nature in Visionary Small Enterprise. Business \& Society, 49(3), 512-547.

Kruse, T., Veltri, A. and Branscum, A. (2019). Integrating safety, health and environmental management systems: A conceptual framework for achieving lean enterprise outcomes. Journal of Safety Research, 71, 259-271.

Li, H., Zhao, Y. and Zheng, F. (2020). The framework of an agricultural land-use decision support system based on ecological environmental constraints. Science of The Total Environment, 717, 137-149.

Melnyk, K. and Tsesarsky, F. (2019). Environmental safety of enterprises activity as a precondition for compliance with the required working conditions. Ekoloji, 28, 463-469.

Ormazabal, M., Rich, E., Sarriegi, M. and Viles, E. (2017). Environmental Management Evolution Framework: Maturity Stages and Causal Loops. Organization \& Environment, 30(1), 27-50.

Puppim de Oliveira, A. and Jabbour, C. (2017). Environmental Management, Climate Change, CSR, and Governance in Clusters of Small Firms in Developing Countries: Toward an Integrated Analytical Framework. Business \& Society, 56(1), 130-151.

Ramirez, M., Clarke, I. and Klerkx, L. (2018). Analysing intermediary organisations and their influence on upgrading in emerging agricultural clusters. Environment and Planning A: Economy and Space, 50(6), $1314-1335$.

Samborskyi, O., Isaia, O., Hnatenko, I., Parkhomenko, O., Rubezhanska V. and Yershova, O. (2020). Modeling of foreign direct investment impact on economic growth in a free market. Accounting, 6(5), 705-712.

State Statistics Service of Ukraine (2019). Agriculture of Ukraine 2018. Kyiv, Ukraine. Available at: http://www.ukrstat.gov.ua [Accessed: 21 August 2020].

Tang, Sh.; Tang, C. and Wing-Hung Lo, C. (2015). Public Participation and Environmental Impact Assessment in Mainland China and Taiwan: Political Foundations of Environmental Management. The Journal of Development Studies, 41(1), 1-32. 